\title{
Multi-quasi-synchronization of coupled fractional-order neural networks with delays via pinning impulsive control
}

\author{
Xiaoli Ruan ${ }^{1}$ and Ailong $\mathrm{Wu}^{1,2,3 *}$
}

\section{"Correspondence:}

hbnuwu@yeah.net

${ }^{1}$ College of Mathematics and

Statistics, Hubei Normal University,

Huangshi, 435002, China

${ }^{2}$ Institute for Information and

System Science, Xi'an Jiaotong University, Xi'an, 710049, China

Full list of author information is

available at the end of the article

\section{算 Springer}

\begin{abstract}
We investigate the collective dynamics of multi-quasi-synchronization of coupled fractional-order neural networks with delays. Using the pinning impulsive strategy, we design a novel controller to pin the coupled networks to realize the multi-quasi-synchronization. Based on the comparison principle and mathematical analysis, we derive some novel criteria of the multi-quasi-synchronization. Moreover, we discuss the effects of coupling strength and pinning control matrix. Finally, some simulation examples show the effectiveness of the presented results.
\end{abstract}

Keywords: multi-quasi-synchronization; fractional-order neural networks; comparison principle; pinning impulsive control

\section{Introduction}

In the past decades, fractional-order derivatives have been drawn wide attention. Compared with integer-order derivatives, it has a greater advantage in describing the memory and hereditary properties of manifold materials and processes (see [1-3]). It is better to describe many practical problems by fractional-order dynamical systems instead of integerorder ones. They are extensively applicable in many areas, such as physics, polymer rheology, electrical circuits, and engineering optimization [4-8]. In addition, the fractional differentiation has been extended to the computational methods involved to travelingwave transformation [9-11]. Yang et al. [9] investigated exact traveling-wave solutions of nondifferentiable type with the generalized functions for the local fractional Korteweg-de Vries equation. Exact traveling-wave solution for the local fractional Boussinesq equation in fractal domain was studied in [10]. Yang et al. [11] analyzed the exact travelling-wave solutions for a family of the local fractional two-dimensional Burgers-type equation.

As one of the mostly important collective behaviors of complex dynamic networks, synchronization has been extensively investigated [12-19]. The problem of synchronization of coupled fractional-order neural networks has been well studied. For instance, Ma and Zhang [20] showed that two coupled networks can achieve a hybrid synchronization by some proper conditions. Later, Ma and Zhang [21] studied a new hybrid projective synchronization of two different-size coupled fractional-order complex networks. A generalized chaos synchronization of coupled Mathieu-Van der Pol and coupled Duffing-Van der Pol systems using fractional-order derivatives was shown in [22]. In addition, a general

(c) The Author(s) 2017. This article is distributed under the terms of the Creative Commons Attribution 4.0 International License (http://creativecommons.org/licenses/by/4.0/), which permits unrestricted use, distribution, and reproduction in any medium, provided you give appropriate credit to the original author(s) and the source, provide a link to the Creative Commons license, and indicate if changes were made. 
chaotic synchronization of fractional chaotic maps based on the stability condition was investigated in [23].

Various control techniques have been adopted to realize synchronization, such as pinning control [24], feedback control [25], impulsive control [26, 27], adaptive control [28], intermittent control [29], and so on. Jajarmi et al. [28] analyzed a hyperchaotic financial system and its adaptive control and synchronization. However, in real world, it is too costly and impractical if all the nodes are controlled [30]. To reduce the control cost, it is extremely effective to control a complex network by controlling a certain time and pinning part of nodes [31,32]. Recently, some researchers have combined the advantages of pinning control and impulsive control to investigate the synchronization problem. He et al. [33] studied the synchronization of coupled delayed dynamical networks via pinning impulsive control. However, the above results are only concerned with complete synchronization. Due to the external disturbances and internal uncertainty in networks, it is more realistic that for nodes in each subgroup, only a quasi-synchronization can be achieved. Just a few papers investigated multi-quasi-synchronization of coupled networks [34, 35]. To the best of our knowledge, there are no results on the multi-quasi-synchronization of coupled fractional-order networks with delays via pinning impulsive control. Motivated by the above discussion, in this paper, we investigate the multi-quasi-synchronization of coupled fractional-order neural networks with delays via pinning impulsive control.

The main contributions of this paper can be summarized as follows:

- Developing the multisynchronization concept. Multi-quasi-synchronization generalizes quasi-synchronization, cluster synchronization, etc.

- A new pinning impulsive control method is proposed to deal with the multi-quasi-synchronization problem.

- By using the comparison principle and inequality techniques some weaker conservative conditions are derived.

This paper is composed as follows. Section 2 describes some preliminaries. The main results are presented in Section 3. Some examples are given in Section 4. Finally, some conclusions are drawn in Section 5.

\section{Preliminaries and model description}

\subsection{Preliminaries about fractional-order calculus}

In the following, we introduce some notation, definitions, and lemmas.

The superscript $T$ represents the transpose. $\mathbb{R}^{n}$ denotes the $n$-dimensional Euclidean space, $\mathbb{R}^{n \times n}$ is the set of $n \times n$ real matrices, $\mathbb{R}^{+}$and $\mathbb{Z}^{+}$denote the sets of nonnegative real numbers and positive integers, respectively, and $\sharp \mathfrak{D}$ indicates the number of elements of a finite set $\mathfrak{D}$. For any vector $d \in \mathbb{R}^{n}$ and constant $\sigma_{0}>0, \mathfrak{M}\left(d, \sigma_{0}\right)=\{x \mid\|x-d\|<$ $\left.\sigma_{0}\right\}$ denotes the set of vectors whose distance to $d$ is less than $\sigma_{0} . A \otimes B$ represents the Kronecker product of matrices $A$ and $B$. We write that a real symmetric matrix $Y>0(Y<$ $0, Y \geq 0, Y \leq 0$ ) if $Y$ is positive definite (negative definite, positive semidefinite, negative semidefinite). For any matrix $A, \lambda_{\max }(A)$ denotes its maximum eigenvalue, and its spectral norm is defined as $\|A\|=\left(\lambda_{\max }\left(A^{T} A\right)\right)^{\frac{1}{2}}$. 
Definition $2.1([1]) \Gamma(\cdot)$ denotes the gamma function. The Caputo fractional derivative of order $\alpha>0$ for a function $f(t)$ is defined as

$$
{ }^{c} D_{t_{0}, t}^{\alpha} f(t)=\frac{1}{\Gamma(n-\alpha)} \int_{t_{0}}^{t}(t-s)^{(n-\alpha-1)} f^{(n)}(s) d s, \quad t \geq t_{0},
$$

where $n-1<\alpha<n, n \in \mathbb{Z}^{+}$.

Definition 2.2 ([1]) The fractional integral of order $\alpha>0$ for a function $f(t)$ is defined as

$$
I_{t_{0}, t}^{\alpha} f(t)=\frac{1}{\Gamma(\alpha)} \int_{t_{0}}^{t}(t-s)^{\alpha-1} f(s) d s, \quad t \geq t_{0}
$$

Definition 2.3 ([1]) The one-parameter Mittag-Leffler function is defined as

$$
E_{\alpha}(z)=\sum_{k=0}^{+\infty} \frac{z^{k}}{\Gamma(k \alpha+1)}
$$

where $\alpha>0, z \in \mathbb{C}$.

The two-parameter Mittag-Leffler function is defined as

$$
E_{\alpha, \beta}(z)=\sum_{k=0}^{+\infty} \frac{z^{k}}{\Gamma(k \alpha+\beta)}
$$

where $\alpha>0, \beta>0$, and $z \in \mathbb{C}$.

\subsection{Model}

In this paper, we consider a delayed fractional-order neural network consisting of $N$ identical nodes, which is described by

$$
{ }^{c} D_{t_{0}, t}^{\alpha} x_{i}(t)=-A x_{i}(t)+B f_{i}\left(x_{i}(t)\right)+C f_{i}\left(x_{i}(t-\tau)\right)+\sum_{j=1}^{N} G_{i j} \Gamma x_{j}(t)+J,
$$

where $i=1,2, \ldots, N, N \geq 2$ is the number of subnetworks; $x_{i}(t)=\left(x_{i 1}(t), x_{i 2}(t), \ldots, x_{i n}(t)\right)^{T}$ denotes the state vector of the $i$ th neural network; $A=\operatorname{diag}\left\{a_{1}, a_{2}, \ldots, a_{n}\right\}>0$ represents the self-feedback term of the $j$ th neuron; $B=\left(b_{p q}\right)_{n \times n}$ and $C=\left(c_{p q}\right)_{n \times n}(p, q=1,2, \ldots, n)$ are the connection weight matrix and the delayed connection matrix, respectively; $f_{i}\left(x_{i}(t)\right)=$ $\left(f_{i 1}\left(x_{i 1}(t)\right), f_{i 2}\left(x_{i 1}(t)\right), \ldots, f_{i n}\left(x_{i n}(t)\right)\right)^{T}$ where $f_{i j}(\cdot), j=1,2, \ldots, n$, is the activation function; $\tau$ represents the transmission delay; $G=\left(G_{i j}\right)_{N \times N}$ is the coupling matrix defined as follows: if there is a link from node $j$ to node $i$, then $G_{i j}>0$ and otherwise $G_{i j}=0$, the diagonal elements are defined as $G_{i i}=\sum_{j=1, j \neq i}^{N}-G_{i j} ; \Gamma=\operatorname{diag}\left\{\gamma_{1}, \gamma_{2}, \ldots, \gamma_{n}\right\}$, which represents the inner coupling matrix; and $J=\left(J_{1}, \ldots, J_{N}\right)$ is a constant external input vector.

Assumption 2.1 The activation functions $f_{i}(\cdot)$ are continuous, and there exist $\Pi_{i}>0$ such that, for any vectors $x, y$, we have

$$
\left|f_{i}(x)-f_{i}(y)\right| \leq \Pi_{i}|x-y|
$$


Suppose that if for any initial state $x(s)=\left(x_{1}^{T}(s), x_{2}^{T}(s), \ldots, x_{N}^{T}(s)\right)^{T}$ with $x_{i}(s) \in$ $C([-\tau, 0], \mathbb{R}), i=1,2, \ldots, N$, there exists a solution $s(t)$ for any initial condition, then all trajectories of network nodes fulfill $\lim _{t \rightarrow \infty}\left\|x_{i}(t)-s(t)\right\|=0, i=1,2, \ldots, N$. Then the network is said to be completely synchronized [24]. Moreover, if for error bound $\sigma>0$, there exists $T>0$ such that, for any initial state and all $t>T,\left\|x_{i}(t)-s(t)\right\|<\sigma, i=1,2, \ldots, N$, then the network is said to be uniformly quasi-synchronized [36].

Next, we give the definition of the multi-quasi-synchronization.

Definition 2.4 ([34]) For any complex network with $N$ nodes, let $\left\{\mathcal{C}_{1}, \mathcal{C}_{2}, \ldots, \mathcal{C}_{m}\right\}$ be a disjoint division of the node set, that is, $\sum_{k=1}^{m} \mathcal{C}_{k}=\{1,2, \ldots, N\}, \mathcal{C}_{k}=\left\{l_{k 1}, l_{k 2}, \ldots\right\}$, and $\mathcal{C}_{k} \cap \mathcal{C}_{u}=\emptyset$ for $k \neq u$. Suppose that there exists a series of reference solution $\left\{s_{1}(t), s_{2}(t), \ldots, s_{m}(t)\right\}$. The network is said to be multi-quasi-synchronized with an error vector $\delta=\left\{\delta_{1}, \delta_{2}, \ldots, \delta_{m}\right\}^{T}>0$ under any initial condition if for any small enough constant $\varepsilon>0$, there exists $T$ such that, for all $t>T$, the nodes satisfy $x_{i}(t) \in \mathfrak{M}\left(s_{k}(t), \sigma_{k}\right)$ for $i \in \mathcal{C}_{k}$, and $\mathfrak{M}\left(s_{k}(t), \sigma_{k}\right) \neq \mathfrak{M}\left(s_{u}(t), \bar{\sigma}_{u}\right)$ for $u \neq k$.

Remark 2.1 $\sharp \mathcal{C}_{k}=\zeta_{k}$ means that the group $\mathcal{C}_{k}$ has $\zeta_{k}$ nodes and $\zeta_{k} \neq 0$.

Remark 2.2 From Definition 2.4 we know that, for all of the nodes in group $\mathcal{C}_{k}, s_{k}(t)$ is the shared reference trajectory.

The desired trajectory of $s_{\tilde{i}}(t)$ satisfies

$$
{ }^{c} D_{t_{0}, t}^{\alpha} s_{\tilde{i}}(t)=-A s_{\tilde{i}}(t)+B f_{\tilde{i}}\left(s_{\tilde{i}}(t)\right)+C f_{\tilde{i}}\left(s_{\tilde{i}}(t-\tau)+J,\right.
$$

where $s_{\tilde{i}}(t)=s_{k}(t)$ if $x_{i}(t) \in \mathfrak{M}\left(s_{k}(t), \sigma_{k}\right), i=1,2, \ldots, N, k=1,2, \ldots, m$. Define the error signal $e_{i}(t)=x_{i}(t)-s_{\tilde{i}}(t), i=1,2, \ldots, N$. The pinning impulsive controller is designed as

$$
u_{i}(t)= \begin{cases}\sum_{h=1}^{+\infty} \theta_{k} e_{i}(t) \delta\left(t-t_{h}\right), & i \in \mathfrak{D}_{k}\left(t_{h}\right), \sharp \mathfrak{D}_{k}\left(t_{h}\right)=\omega_{k}, \\ 0, & i \notin \mathfrak{D}_{k}\left(t_{h}\right),\end{cases}
$$

where $t_{h}(h=0,1,2, \ldots)$ are the impulsive instants satisfying $0=t_{0}<t_{1}<\cdots<t_{h}<\cdots$, and $\lim _{t_{h} \rightarrow+\infty} t_{h}=+\infty, \delta(\cdot)$ is the Dirac impulsive function, and $\theta_{k}$ is the impulsive gain. Let $\sum_{k=1}^{m} \mathfrak{D}_{k}\left(t_{h}\right)=\left\{\mathfrak{D}_{1}\left(t_{h}\right), \mathfrak{D}_{2}\left(t_{h}\right), \ldots, \mathfrak{D}_{m}\left(t_{h}\right)\right\} \subset\left\{\mathcal{C}_{1}, \mathcal{C}_{2}, \ldots, \mathcal{C}_{m}\right\} \subset\{1,2, \ldots, N\}$ denote the set of pinned nodes at $t=t_{h}$, and let $0<\omega_{k} \leq \zeta_{k}, k=1,2, \ldots, m$, that is, $\mathfrak{D}_{k}\left(t_{h}\right)$ is the subset of $\mathcal{C}_{k}$ indicating the set of pinned nodes at $t=t_{h}$. We also assume that the error vector $e_{i 1} \geq$ $e_{i 2} \geq \cdots \geq e_{i n}$. Under the proposed impulsive control (4), the error system is formulated as

$$
\left\{\begin{array}{l}
{ }^{c} D_{t_{0}, t}^{\alpha} e_{i}(t)=-A e_{i}(t)+B \bar{f}_{i}\left(e_{i}(t)\right)+C \bar{f}_{i}\left(e_{i}(t-\tau)\right)+\sum_{j=1}^{N} G_{i j} \Gamma e_{j}(t), \quad t \neq t_{h}, \\
e_{i}\left(t_{h}^{+}\right)=e_{i}\left(t_{h}^{-}\right)+\theta_{k} e_{i}\left(t_{h}^{-}\right), \quad i \in \sum_{k=1}^{m} \mathfrak{D}_{k}\left(t_{h}\right), \\
e_{i}\left(t_{h}^{+}\right)=e_{i}\left(t_{h}^{-}\right), \quad i \notin \sum_{k=1}^{m} \mathfrak{D}_{k}\left(t_{h}\right),
\end{array}\right.
$$

where $h=0,1,2, \ldots, \bar{f}_{i}\left(e_{i}(t)\right)=f_{i}\left(x_{i}(t)\right)-f_{\tilde{i}}\left(s_{\tilde{i}}(t)\right), \bar{f}_{i}\left(e_{i}(t-\tau)\right)=f_{i}\left(x_{i}(t-\tau)\right)-f_{\tilde{i}}\left(s_{\tilde{i}}(t-\tau)\right)$, $e_{i}\left(t_{h}^{+}\right)=\lim _{t \rightarrow t_{h}^{+}} e_{i}(t)$, and $x_{i}\left(t_{h}^{-}\right)=x_{i}\left(t_{h}\right)$. 
The initial condition of (5) is defined as

$$
e_{i}(s)=\phi_{i}(s), \quad \tau \leq s \leq 0,
$$

where $\phi_{i}(s) \in C\left([-\tau, 0], \mathbb{R}^{n}\right), i=1,2, \ldots, N$.

Definition 2.5 The pinning ratio at $t=t_{h}$ is defined as

$$
\frac{\sum_{i \in \mathfrak{D}_{k}\left(t_{h}\right)} e_{i}^{T}\left(t_{h}^{-}\right) e_{i}\left(t_{h}^{-}\right)}{\sum_{i \in \mathcal{C}_{k}} e_{i}^{T}\left(t_{h}^{-}\right) e_{i}\left(t_{h}^{-}\right)}=\eta_{k}
$$

The pinning ratio is time-varying and related to impulsive instants. We will determine a lower bound of $\eta_{k}$.

Lemma 2.1 ([37]) Let $x(t) \in \mathbb{R}^{n}$ be a continuous and differentiable vector function. Then, for any time instant $t \geq t_{0}$, we have the relationship

$$
\frac{1}{2}{ }^{c} D_{t_{0}, t}^{\alpha}\left(x^{T}(t) P x(t)\right) \leq x^{T}(t) P^{c} D_{t_{0}, t}^{\alpha} x(t)
$$

for all $\alpha \in(0,1)$, where $P \in \mathbb{R}^{n \times n}$ is a constant symmetric positive definite matrix.

Lemma 2.2 ([38]) For any vector $x, y \in \mathbb{R}^{n}$, scalar $\epsilon>0$, and positive definite matrix $Q \in$ $\mathbb{R}^{n \times n}$, we have the inequality

$$
2 x^{T} y \leq \epsilon x^{T} Q x+\epsilon^{-1} y^{T} Q^{-1} y \text {. }
$$

Lemma 2.3 ([39]) Consider the system with time delay

$$
\begin{cases}{ }^{c} D_{t_{0}, t}^{\alpha} V(t) \leq-a V(t)+b V(t-\tau), & t>0, \\ V(s)=\Psi(s), & s \in[-\tau, 0]\end{cases}
$$

and the linear fractional-order differential system with time delay

$$
\begin{cases}{ }^{c} D_{t_{0}, t}^{\alpha} W(t)=-a W(t)+b W(t-\tau), & t>0, \\ W(s)=\Psi(s), & s \in[-\tau, 0]\end{cases}
$$

where $V(t), W(t) \in \mathbb{R}$ are continuous everywhere except at some points $t_{k}, k=1,2, \ldots$, and $\Psi(s) \geq 0$ is continuous in $[-\tau, 0]$. If $a>0$ and $b>0$, then $V(t) \leq W(t), t \in[0,+\infty)$.

Inspired by Lemma 2.3, we can get the following lemma.

Lemma 2.4 Consider the system with time delay

$$
\begin{cases}{ }^{c} D_{t_{0}, t}^{\alpha} V_{k}(t) \leq-K_{1} V_{k}(t)+K_{2} V_{k}(t-\tau), & t>0, i \in \mathcal{C}_{k}, \\ V_{k}(s)=\Phi_{k}(s), & s \in[-\tau, 0]\end{cases}
$$


and the linear fractional-order differential system with time delay

$$
\begin{cases}{ }^{c} D_{t_{0}, t}^{\alpha} W_{k}(t)=-K_{1} W_{k}(t)+K_{2} W_{k}(t-\tau), & t>0, i \in \mathcal{C}_{k}, \\ W_{k}(s)=\Phi_{k}(s), & s \in[-\tau, 0],\end{cases}
$$

where $V_{k}(t), W_{k}(t) \in \mathbb{R}^{n}$ are continuous everywhere except at some point $t_{h}, h=1,2, \ldots$, and $\Phi_{k}(s) \geq 0$ is continuous in $[-\tau, 0]$. If $K_{1}>0$ and $K_{2}>0$, then $V_{k}(t) \leq W_{k}(t), t \in[0,+\infty)$.

Proof For system (7) and for any $i \in \mathcal{C}_{k}$, there exists a nonnegative function $m_{k}(t)$ such that

$$
\begin{cases}{ }^{c} D_{t_{0}, t}^{\alpha} V_{k}(t)=-K_{1} V_{k}(t)+K_{2} V_{k}(t-\tau)-m_{k}(t), & t>0, \\ V_{k}(s)=\Phi_{k}(s), & s \in[-\tau, 0]\end{cases}
$$

Let $l_{1}=\left[\frac{t_{1}}{\tau}\right]+1$, where $\left[\frac{t_{1}}{\tau}\right]$ stands for the greatest integer smaller than $\frac{t_{1}}{\tau}$. Obviously, $\left[0, t_{1}\right) \subseteq\left[0, l_{1} \tau\right)$, and from [40], we know that (9) has a unique solution expressed by $V_{k}(t)=$ $V_{j k}(t)$

$$
V_{j k}(t)=\lambda_{j k} E_{\alpha, 1}\left(-K_{1} t^{\alpha}\right)+\int_{0}^{t}(t-s)^{\alpha-1} E_{\alpha, \alpha}\left(-K_{1}(t-s)^{\alpha}\right) \Omega_{j k} d s, \quad t \in[(j-1) \tau, j \tau],
$$

where $\lambda_{j k}$ is s constants, $j=1,2, \ldots, l_{1}$. When $j=1, V_{0 k}(t)=\Phi_{k}(t)$, and $\Omega_{j k}$ is represented as

$$
\Omega_{j k}(t)= \begin{cases}K_{2} V_{0 k}(t-\tau)-m_{k}(t), & 0<t \leq \tau, \\ K_{2} V_{1 k}(t-\tau)-m_{k}(t), & \tau<t \leq 2 \tau, \\ \vdots & \\ K_{2} V_{\left(l_{1}-1\right) k}(t-\tau)-m_{k}(t), & \left(l_{1}-1\right) \tau<t \leq l_{1} \tau .\end{cases}
$$

From [41] we know that both $t^{\alpha-1}$ and $E_{\alpha, \alpha}\left(-a t^{\alpha}\right)$ are nonnegative functions. Due to $V_{k}(t)=V_{j k}(t)$ and $m_{k}(t)>0$, from (10) and (11) we have

$$
\begin{aligned}
V_{j k}(t) \leq & \lambda_{j k} E_{\alpha, 1}\left(-K_{1} t^{\alpha}\right) \\
& +\int_{0}^{t}(t-s)^{\alpha-1} E_{\alpha, \alpha}\left(-K_{1}(t-s)^{\alpha}\right) K_{2} V_{j k}(s-\tau) d s, \quad t \in[(j-1) \tau, j \tau] .
\end{aligned}
$$

At the same time,

$$
\begin{aligned}
W_{j k}(t)= & \lambda_{j k} E_{\alpha, 1}\left(-K_{1} t^{\alpha}\right) \\
& +\int_{0}^{t}(t-s)^{\alpha-1} E_{\alpha, \alpha}\left(-K_{1}(t-s)^{\alpha}\right) K_{2} W_{j k}(s-\tau) d s, \quad t \in[(j-1) \tau, j \tau] .
\end{aligned}
$$

When $l_{1}=1$ and $t \in[0, \tau]$, we have $t-\tau \in[-\tau, 0]$ and $V_{k}(t-\tau)=W_{k}(t-\tau)=\Phi_{k}(t-\tau)$. From (12) and (13) we have

$$
V_{k}(t) \leq \lambda_{k} E_{\alpha, 1}\left(-K_{1} t^{\alpha}\right)+\int_{0}^{t}(t-s)^{\alpha-1} E_{\alpha, \alpha}\left(-K_{1}(t-s)^{\alpha}\right) K_{2} \Phi_{k}(s-\tau) d s=W_{k}(t)
$$

and so $V_{k}(t) \leq W_{k}(t)$ for $l_{1}=1$. 
Next, suppose that $V_{k}(t) \leq W_{k}(t), k=1,2, \ldots, m$, for $t \in\left[\left(l_{1}-1\right) \tau, l_{1} \tau\right]$. Then we have $V_{j k}(t) \leq W_{j k}(t), j=1,2, \ldots, l_{1}$.

Now, we will prove that this also holds for $l_{1}+1$. If $t \in\left[l_{1} \tau,\left(l_{1}+1\right) \tau\right]$, then (12) can be represented as

$$
\begin{aligned}
V_{k}(t)= & \int_{0}^{t}(t-s)^{\alpha-1} E_{\alpha, \alpha}\left(-K_{1}(t-s)^{\alpha}\right) K_{2} V_{k}(s-\tau) d s \\
& +\lambda_{\left(l_{1}+1\right) k} E_{\alpha, 1}\left(-K_{1} t^{\alpha}\right) \\
= & \int_{0}^{\tau}(t-s)^{\alpha-1} E_{\alpha, \alpha}\left(-K_{1}(t-s)^{\alpha}\right) K_{2} V_{k}(s-\tau) d s \\
& +\sum_{j=2}^{l_{1}} \int_{(j-1) \tau}^{j \tau}(t-s)^{\alpha-1} E_{\alpha, \alpha}\left(-K_{1}(t-s)^{\alpha}\right) K_{2} V_{j k}(s-\tau) d s \\
& +\int_{l_{1} \tau}^{t}(t-s)^{\alpha-1} E_{\alpha, \alpha}\left(-K_{1}(t-s)^{\alpha}\right) K_{2} V_{\left(l_{1}+1\right) k}(s-\tau) d s \\
& +\lambda_{\left(l_{1}+1\right) k} E_{\alpha, 1}\left(-K_{1} t^{\alpha}\right) .
\end{aligned}
$$

System (13) can be represented as

$$
\begin{aligned}
W_{k}(t)= & \int_{0}^{t}(t-s)^{\alpha-1} E_{\alpha, \alpha}\left(-K_{1}(t-s)^{\alpha}\right) K_{2} W_{k}(s-\tau) d s \\
& +\lambda_{\left(l_{1}+1\right) k} E_{\alpha, 1}\left(-K_{1} t^{\alpha}\right) \\
= & \int_{0}^{\tau}(t-s)^{\alpha-1} E_{\alpha, \alpha}\left(-K_{1}(t-s)^{\alpha}\right) K_{2} W_{k}(s-\tau) d s \\
& +\sum_{j=2}^{l_{1}} \int_{(j-1) \tau}^{j \tau}(t-s)^{\alpha-1} E_{\alpha, \alpha}\left(-K_{1}(t-s)^{\alpha}\right) K_{2} W_{j k}(s-\tau) d s \\
& +\int_{l_{1} \tau}^{t}(t-s)^{\alpha-1} E_{\alpha, \alpha}\left(-K_{1}(t-s)^{\alpha}\right) K_{2} W_{\left(l_{1}+1\right) k}(s-\tau) d s \\
& +\lambda_{\left(l_{1}+1\right) k} E_{\alpha, 1}\left(-K_{1} t^{\alpha}\right)
\end{aligned}
$$

for $s \in\left[l_{1} \tau, t\right], s-\tau \in\left[\left(l_{1}-1\right) \tau, t-\tau\right] \subseteq\left[\left(l_{1}-1\right) \tau, l_{1} \tau\right]$. According to the assumption $V_{k}(t) \leq$ $W_{k}(t)$, we have $V_{k}(s-\tau) \leq W_{k}(s-\tau)$, and from (14) and (15) we get that $V_{k}(t) \leq W_{k}(t)$, $t \in\left[l_{1} \tau,\left(l_{1}+1\right) \tau\right]$.

Denote $l_{2}=\left[\frac{t_{2}}{\tau}\right]+1$. Then, obviously, $\left[t_{1}, t_{2}\right) \subseteq\left[t_{1}, l_{2} \tau\right)$. The initial conditions of (9) and (10) can be represented as follows:

$$
\begin{aligned}
& V_{k}(s)=\Phi_{k}(s), \quad s \in\left[t_{1}-\tau, t_{1}\right), \\
& W_{k}(s)=\Phi_{k}(s), \quad s \in\left[t_{1}-\tau, t_{1}\right) .
\end{aligned}
$$

Similarly to the proof for $t \in\left[0, t_{1}\right)$, we get that $V_{k}(t) \leq W_{k}(t)$ for $t \in\left[t_{1}, t_{2}\right)$. 
So, dividing the $[0,+\infty)$ into a union of all subsets $\left[0, t_{1}\right) \cup\left[t_{1}, t_{2}\right) \cup \cdots \cup\left[t_{k-1}, t_{k}\right) \cup \cdots$, we prove that $V_{k}(t) \leq W_{k}(t)$ for $t \in[0,+\infty)$.

\section{Main results}

In this section, we derive several synchronization criteria. Under the impulsive control, in any group $\mathcal{C}_{k} \in\left\{\mathcal{C}_{1}, \mathcal{C}_{2}, \ldots, \mathcal{C}_{m}\right\}$, for $i \in \mathcal{C}_{k}$, the network (1) is able to synchronize with $s_{k}(t) \in\left\{s_{1}(t), \ldots, s_{m}(t)\right\}$ and realize multi-quasi-synchronization.

Theorem 3.1 For any $i \in \mathcal{C}_{k}, k=1,2, \ldots, m$, system (5) can realize multi-quasisynchronization by pinning impulsive control (4) if Assumption 2.1 holds and there exist positive definite matrix $P \in \mathbb{R}_{n \times n}>0$ and diagonal matrices $\Sigma_{1} \in \mathbb{R}^{n \times n}>0$ and $\Sigma_{2} \in \mathbb{R}^{n \times n}>0$ such that

$$
\begin{aligned}
& \left(\begin{array}{cc}
G & 0 \\
0 & P \Gamma
\end{array}\right)<0, \\
& \Lambda=P A+A^{T} P-P B \Sigma_{1}^{-1} B^{T} P-\Pi_{i}^{T} \Sigma_{1} \Pi_{i}-P B \Sigma_{2}^{-1} C^{T} P \geq K_{1} P>0, \\
& \Pi_{i}^{T} \Sigma_{2} \Pi_{i} \leq K_{2} P, \\
& \left(1+\theta_{k}\right)^{2} \eta_{k} \frac{\lambda_{\max }(P)}{\lambda_{\min }(P)}+\left(1-\eta_{k}\right) \frac{\lambda_{\max }(P)}{\lambda_{\min }(P)} \leq \rho_{k} \in(0,1),
\end{aligned}
$$

where $K_{1}>0, K_{2}>0, K_{1}>\sqrt{2} K_{2}$, and $\sqrt{\frac{\lambda_{\max }(P) \varepsilon}{\lambda_{\min }(P)}}<\sigma_{k}$.

Proof Let us consider the Lyapunov function $V_{k}(t)=\sum_{i \in \mathcal{C}_{k}} e_{i}^{T}(t) P e_{i}(t)$.

For $t \in\left[t_{h-1}, t_{h}\right), h=0,1,2, \ldots$, from Lemma 2.1 we have

$$
\begin{aligned}
{ }^{c} D_{t_{0}, t}^{\alpha} V_{k}(t) \leq & 2 \sum_{i \in \mathcal{C}_{k}} e_{i}^{T}(t) P^{c} D_{t_{0}, t}^{\alpha} e_{i}(t) \\
= & 2 \sum_{i \in \mathcal{C}_{k}} e_{i}^{T}(t) P\left(-A e_{i}(t)+B \bar{f}_{i}\left(e_{i}(t)\right)+C \bar{f}_{i}\left(e_{i}(t-\tau)\right)\right. \\
& \left.+\sum_{j \in \mathcal{C}_{k}} G_{i j} \Gamma e_{j}(t)\right) \\
= & 2 \sum_{i \in \mathcal{C}_{k}} e_{i}^{T}(t)(-P A) e_{i}(t)+2 \sum_{i \in \mathcal{C}_{k}} e_{i}^{T}(t) P B \bar{f}_{i}\left(e_{i}(t)\right) \\
& +2 \sum_{i \in \mathcal{C}_{k}} e_{i}^{T}(t) P C \bar{f}_{i}\left(e_{i}(t-\tau)\right) \\
& +2 \sum_{i \in \mathcal{C}_{k}} e_{i}^{T}(t) P \sum_{j \in \mathcal{C}_{k}} G_{i j} \Gamma e_{j}(t) .
\end{aligned}
$$

Clearly,

$$
2 \sum_{i \in \mathcal{C}_{k}} e_{i}^{T}(t) P A e_{i}(t) \leq \sum_{i \in \mathcal{C}_{k}} e_{i}^{T}(t)\left(-P A-A^{T} P\right) e_{i}(t)
$$


By Assumption 2.1 and Lemma 2.2, for positive definite diagonal matrices $\Sigma_{1}$ and $\Sigma_{2}$, we obtain

$$
\begin{aligned}
& \sum_{i \in \mathcal{C}_{k}} 2 e_{i}^{T}(t) P B \bar{f}_{i}\left(e_{i}(t)\right) \leq \sum_{i \in \mathcal{C}_{k}} e_{i}^{T}(t) P B \bar{f}_{i}\left(e_{i}(t)+\sum_{i \in \mathcal{C}_{k}} \bar{f}_{i}^{T}\left(e_{i}(t)\right) B^{T} P e_{i}(t)\right. \\
& \leq \sum_{i \in \mathcal{C}_{k}} e_{i}^{T}(t) P B \Sigma_{1}^{-1} B^{T} P e_{i}(t) \\
& +\sum_{i \in \mathcal{C}_{k}} \bar{f}_{i}^{T}\left(e_{i}(t)\right) \Sigma_{1} \bar{f}_{i}\left(e_{i}(t)\right) \\
& \leq \sum_{i \in \mathcal{C}_{k}} e_{i}^{T}(t) P B \Sigma_{1}^{-1} B^{T} P e_{i}(t) \\
& +\sum_{i \in \mathcal{C}_{k}} e_{i}^{T}(t) \Pi_{i}^{T} \Sigma_{1} \Pi_{i} e_{i}(t) \\
& =\sum_{i \in \mathcal{C}_{k}} e_{i}^{T}(t)\left(P B \Sigma_{1}^{-1} B^{T} P+\Pi_{i}^{T} \Sigma_{1} \Pi_{i}\right) e_{i}(t) \\
& \sum_{i \in \mathcal{C}_{k}} 2 e_{i}^{T}(t) P C \bar{f}_{i}\left(e_{i}(t-\tau)\right) \leq \sum_{i \in \mathcal{C}_{k}} e_{i}^{T}(t) P C \bar{f}_{i}\left(e_{i}(t-\tau)\right) \\
& +\sum_{i \in \mathcal{C}_{k}} \bar{f}_{i}^{T}\left(e_{i}(t-\tau)\right) C^{T} P e_{i}(t) \\
& \leq \sum_{i \in \mathcal{C}_{k}} e_{i}^{T}(t) P C \Sigma_{2}^{-1} C^{T} P e_{i}(t) \\
& +\sum_{i \in \mathcal{C}_{k}} \bar{f}_{i}^{T}\left(e_{i}(t-\tau)\right) \Sigma_{2} \bar{f}_{i}\left(e_{i}(t-\tau)\right) \\
& \leq \sum_{i \in \mathcal{C}_{k}} e_{i}^{T}(t) P C \Sigma_{2}^{-1} C^{T} P e_{i}(t) \\
& +\sum_{i \in \mathcal{C}_{k}} e_{i}^{T}(t-\tau) \Pi_{i}^{T} \Sigma_{2} \Pi_{i} e_{i}(t-\tau)
\end{aligned}
$$

From (16) it follows that

$$
\sum_{i \in \mathcal{C}_{k}} 2 e_{i}^{T}(t) \sum_{j \in \mathcal{C}_{k}} G_{i j} \Gamma e_{j}(t)=2 e^{T}(t)(G \otimes P \Gamma) e(t) \leq 0
$$

Substituting (21)-(24) into (20) yields

$$
\begin{aligned}
D_{t_{0}, t}^{\alpha} V_{k}(t) \leq & \sum_{i \in \mathcal{C}_{k}} e_{i}^{T}(t)\left(-P A-A^{T} P\right) e_{i}(t) \\
& +\sum_{i \in \mathcal{C}_{k}} e_{i}^{T}(t)\left(P B \Sigma_{1}^{-1} B^{T} P+\Pi_{i}^{T} \Sigma_{1} \Pi_{i}\right) e_{i}(t) \\
& +\sum_{i \in \mathcal{C}_{k}} e_{i}^{T}(t-\tau) \Pi_{i}^{T} \Sigma_{2} \Pi_{i} e_{i}(t-\tau)+2 e^{T}(t)(G \otimes P \Gamma) e(t) .
\end{aligned}
$$


From (16)-(18) we have

$$
\begin{aligned}
D_{t_{0}, t}^{\alpha} V_{k}(t) & \leq \sum_{i \in \mathcal{C}_{k}}-e_{i}^{T}(t) K_{1} P e_{i}(t)+e_{i}^{T}(t-\tau) K_{2} P e_{i}(t-\tau) \\
& \leq-K_{1} V_{k}(t)+K_{2} V_{k}(t-\tau) .
\end{aligned}
$$

When $t=t_{h}$, from Definition 2.5 and (19) it follows that

$$
\begin{aligned}
V_{k}\left(t_{h}^{+}\right)= & \sum_{i \in \mathcal{C}_{k}} e_{i}^{T}\left(t_{h}^{+}\right) P e_{i}\left(t_{h}^{+}\right) \\
= & \sum_{i \in \mathfrak{D}_{k}\left(t_{h}\right)} e_{i}^{T}\left(t_{h}^{+}\right) P e_{i}\left(t_{h}^{+}\right)+\sum_{i \notin \mathfrak{D}_{k}\left(t_{h}\right)} e_{i}^{T}\left(t_{h}^{+}\right) P e_{i}\left(t_{h}^{+}\right) \\
= & \left(1+\theta_{k}\right)^{2} \sum_{i \in \mathfrak{D}_{k}\left(t_{h}\right)} e_{i}^{T}\left(t_{h}^{-}\right) P e_{i}\left(t_{h}^{-}\right) \\
& +\sum_{i \notin \mathfrak{D}_{k}\left(t_{h}\right)} e_{i}^{T}\left(t_{h}^{-}\right) P e_{i}\left(t_{h}^{-}\right) \\
\leq & \left(1+\theta_{k}\right)^{2} \lambda_{\max }(P) \sum_{i \in \mathfrak{D}_{k}\left(t_{h}\right)} e_{i}^{T}\left(t_{h}^{-}\right) e_{i}\left(t_{h}^{-}\right) \\
& +\lambda_{\max }(P) \sum_{i \notin \mathfrak{D}_{k}\left(t_{h}\right)} e_{i}^{T}\left(t_{h}^{-}\right) e_{i}\left(t_{h}^{-}\right) \\
\leq & \left(1+\theta_{k}\right)^{2} \lambda_{\max }(P) \sum_{i \in \mathcal{C}_{k}} e_{i}^{T}\left(t_{h}\right) e_{i}\left(t_{h}\right) \\
& +\left(1-\eta_{k}\right) \lambda_{\max }(P) \sum_{i \in \mathcal{C}_{k}} e_{i}^{T}\left(t_{h}\right) e_{i}\left(t_{h}\right) \\
\leq & \left(\left(1+\theta_{k}\right)^{2} \eta_{k} \frac{\lambda_{\max }(P)}{\lambda_{\min }(P)}+\left(1-\eta_{k}\right) \frac{\lambda_{\max }(P)}{\lambda_{\min }(P)}\right) V_{k}\left(t_{h}\right) \\
\leq & \rho_{k} V_{k}\left(t_{h}\right) .
\end{aligned}
$$

Now, consider the system

$$
\begin{cases}D_{t_{0}, t}^{\alpha} W_{k}(t)=-K_{1} W_{k}(t)+K_{2} W_{k}(t-\tau), & t>0, \\ W_{k}(s)=\Phi_{k}(s), & s \in[-\tau, 0]\end{cases}
$$

If $\lim _{t \rightarrow \infty} W_{k}(t)=0$ with $\Phi_{k}(s) \geq 0$, then from Lemma 2.4 we have $\lim _{t \rightarrow \infty} V_{k}(t)=0$ with $\Phi_{k}(s) \geq 0$.

Next, we will show that when $K_{1}>\sqrt{2} K_{2}\left(K_{1}>0, K_{2}>0\right), \lim _{t \rightarrow+\infty} W_{k}(t)=0$ with $\Phi_{k}(s) \geq 0$.

To distinguish the imaginary unit $i$ from the subnetwork subscript $i$, we denote the imaginary unit $i$ by $\hat{i}$.

By Corollary 3 in [42] the characteristic equation of (27) can be written as

$$
\vartheta_{k}^{\alpha}+K_{1}-K_{2} e^{-\vartheta_{k} \tau}=0
$$


If (28) has no purely imaginary roots and $K_{1}>\sqrt{2} K_{2}$, then the zero solution of equation (27) is Lyapunov globally asymptotically stable, that is, $\lim _{t \rightarrow+\infty} W_{k}(t)=0$ with $\Phi_{k}(s)>0$. Suppose that equation (28) has a purely imaginary root $\vartheta_{k}=w_{k} \hat{i}=\left|w_{k}\right|\left(\cos \frac{\pi}{2}+\hat{i} \sin \frac{\pi}{2}\right)$, where $w_{k}$ is a real number; if $w_{k}>0$, then $\vartheta_{k}=w_{k} \hat{i}=\left|w_{k}\right|\left(\cos \frac{\pi}{2}+\hat{i} \sin \frac{\pi}{2}\right)$, and if $w_{k} \leq 0$, then $\vartheta_{k}=w_{k} \hat{i}=\left|w_{k}\right|\left(\cos \frac{\pi}{2}-\hat{i} \sin \frac{\pi}{2}\right)$.

Submitting $\vartheta_{k}=w_{k} \hat{i}$ into equation (28), we obtain

$$
\left(w_{k} \hat{i}\right)^{\alpha}+K_{1}-K_{2} e^{-\tau w_{k} \hat{i}}=0,
$$

that is,

$$
\begin{aligned}
\left|\left(w_{k} \hat{i}\right)^{\alpha}+K_{1}\right|^{2}=\left|K_{2} e^{-\tau w_{k} \hat{i}}\right|^{2}, & \\
\left|w_{k}\right|^{2 \alpha}+2 K_{1} \cos \left(\frac{\alpha \pi}{2}\right)\left|w_{k}\right|^{\alpha}+K_{1}^{2} & =\left|K_{2} \cos w_{k} \tau\right|^{2}+\left|K_{2} \sin w_{k} \tau\right|^{2} \\
\leq & \leq 2\left(K_{2}\right)^{2} .
\end{aligned}
$$

Let

$$
g_{k}\left(x_{k}\right)=x_{k}^{2}+2 K_{1} k \cos \left(\frac{\alpha \pi}{2}\right) x_{k}+\left(K_{1}\right)^{2}-\left(K_{2} \cos w_{k} \tau\right)^{2}-\left(K_{2} \sin w_{k} \tau\right)^{2} .
$$

Then

$$
g_{k}(0) \geq K_{1}^{2}-2\left(K_{1}\right)^{2}
$$

Since $K_{1}>\sqrt{2} K_{2}, K_{1}>0$, and $K_{2}>0$, we have $K_{1}-2\left(K_{2}\right)^{2}>0$ and $g_{k}(0)>0$. We know that $g_{k}$ is a polynomial of order 2 , so that $g\left(\left|w_{k}\right|^{\alpha}\right)>0$, a contradiction with (29). This means that equation (29) has no solution, which implies that equation (28) has no purely imaginary roots, that is, $\lim _{t \rightarrow+\infty} V_{k}(t)=0$.

So for any $\varepsilon>0$, there exists $T_{k}$ such that, for all $t>T_{k}$,

$$
V_{k}(t)<\lambda_{\max }(P) \varepsilon, \quad t>T_{k}
$$

that is,

$$
\lambda_{\min }(P)\left\|e_{i}(t)\right\|^{2}<\lambda_{\max }(P) \varepsilon,
$$

and thus

$$
\left\|e_{i}(t)\right\| \leq \sqrt{\frac{\lambda_{\max }(P) \varepsilon}{\lambda_{\min }(P)}}<\sigma_{k},
$$

where $i \in \mathcal{C}_{k}$ and $\sum_{k=1}^{m} T_{k}=\left\{T_{1}, T_{2}, \ldots, T_{N}\right\}$. Therefore, for any arbitrary small positive value $\sigma_{k}>0$, there exits $T=\max \left\{T_{1}, T_{2}, \ldots, T_{N}\right\}$ such that, for all $t>T, 0<\left\|x_{i}(t)-s_{k}(t)\right\| \leq$ $\sigma_{k}, k=1,2, \ldots, m$. The proof is completed. 
Remark 3.1 Theorem 3.1 presents a general result on multi-quasi-synchronization of fractional-order neural networks, and meanwhile the error level is clearly expressed. In the existing literature, very few results have been reported about multi-quasi-synchronization of coupled fractional-order neural networks. Unlike analytical methods in [33-35], model (1) is a fractional-order system rather than the integer-order model in [33-35]. Obviously, the method of analysis and design for integral-order systems cannot be referred to deal with fractional-order systems.

Remark 3.2 Multi-quasi-synchronization contains quasi-synchronization. When $m=1$, there is just one shared reference trajectory, and then the multi-quasi-synchronization degenerates to the quasi-synchronization.

Remark 3.3 Jajarmi et al. [28] solved synchronization problems via adaptive control scheme. In this paper, to achieve pinning impulsive control, we just need to control partial nodes into a bounded neighborhood of its shared reference trajectory. It is possible to pin nodes with low-norm value if the control cost is reachable. In real world, nodes with smaller error norm are preferred for a fast convergence. Then it is better to choose nodes with smaller error norm.

\section{Illustrative examples}

In this section, we provide three numerical examples to substantiate the theoretical results.

Example 4.1 Consider the delayed fractional-order neural network

$$
{ }^{c} D_{t_{0}, t}^{\alpha} x_{i}(t)=-A x_{i}(t)+B f_{i}\left(x_{i}(t)\right)+C f_{i}\left(x_{i}(t-\tau)\right)+\sum_{j=1}^{N} G_{i j} \Gamma x_{j}(t)+J,
$$

where $i=1,2, \tau=1, \alpha=0.98, f_{i}\left(x_{i}(t)\right)=\tanh \left(x_{i}(t)\right)$, taking the networks with two nodes and two neurons in every subnetwork. In more detail, the parameters of the subnetwork are given as

$$
\begin{aligned}
& A=\left(\begin{array}{ll}
1 & 0 \\
0 & 1
\end{array}\right), \quad B=\left(\begin{array}{cc}
5 & -1 \\
-0.5 & 4
\end{array}\right), \quad C=\left(\begin{array}{cc}
0.5 & 0 \\
0 & 1
\end{array}\right), \quad G=\left(\begin{array}{cc}
-5 & 0.2 \\
0.4 & -1
\end{array}\right), \\
& J=\left(\begin{array}{l}
0 \\
0
\end{array}\right) .
\end{aligned}
$$

Let $m=2$ and $\tau=1$. Two shared reference trajectories can be expressed as

$$
{ }^{c} D_{t_{0}, t}^{\alpha} s_{k}(t)=-A s_{k}(t)+B f_{k}\left(s_{k}(t)\right)+C f_{k}\left(s_{k}(t-1)\right)+J, \quad k=1,2,
$$

where $f_{k}\left(s_{k}(t)\right)=\tanh \left(s_{k}(t)\right), k=1,2, \alpha=0.98$.

We choose $\Gamma=\operatorname{diag}(1,1)$, impulsive gain $\theta_{1}=-0.1, \theta_{2}=-0.5, \rho_{1}=0.5, \rho_{2}=0.48, \eta_{1}=$ $0.05, \eta_{2}=0.3$. We can verify that conditions (16)-(19) in Theorem 3.1 hold. By exploiting 


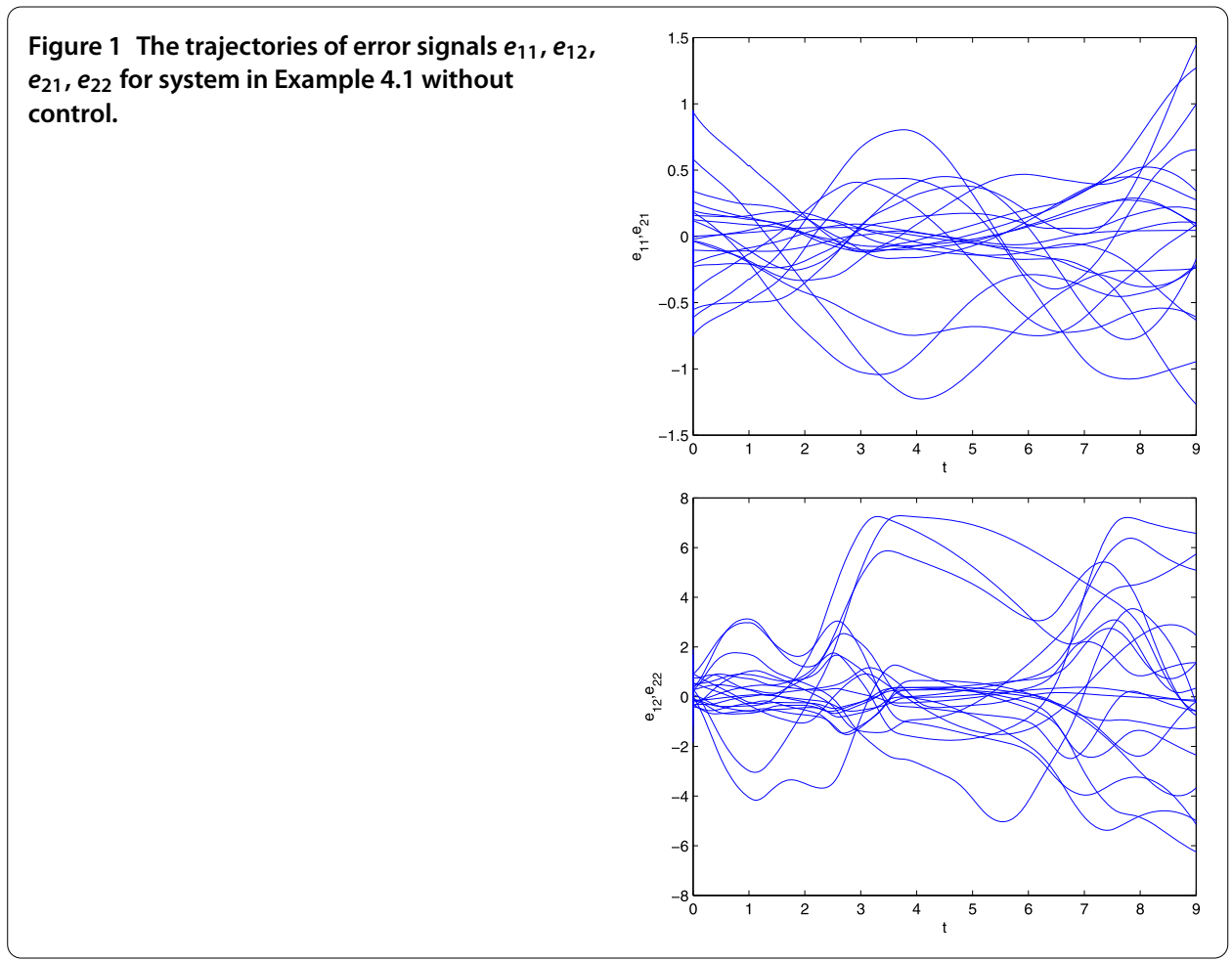

the MATLAB LMI Toolbox we get the matrices

$$
\begin{array}{ll}
P & =\left(\begin{array}{ll}
1.0017 & 0.0022 \\
0.0022 & 1.0945
\end{array}\right), \quad \Sigma_{1}=\left(\begin{array}{cc}
3.0120 & 0.1102 \\
0.1102 & 23.0847
\end{array}\right), \\
\Sigma_{2} & =\left(\begin{array}{ll}
0.4908 & 0.0051 \\
0.0051 & 0.5362
\end{array}\right),
\end{array}
$$

and $\frac{\lambda_{\min }(P)}{\lambda_{\max }(P)}=0.9, \sigma_{1}=0.04, \sigma_{2}=0.21$. Set the pinning ratio $\eta_{k}=0.5$. Then system (33) can achieve multi-quasi-synchronization. Figure 1 shows the disorganized behavior of error signals $e_{i 1}(t)$ and $e_{i 2}(t)(i=1,2)$ without controller. From Figure 1 we can obtain that the whole network exhibits irregular behavior without controller. Based on Figure 2, it shows that the error signals $e_{i 1}(t)$ and $e_{i 2}(t)(i=1,2)$ always converge to different shared reference trajectories with the proposed pinning impulsive control.

Example 4.2 Consider the delayed fractional-order neural network

$$
{ }^{c} D_{t_{0}, t}^{\alpha} x_{i}(t)=-A x_{i}(t)+B f_{i}\left(x_{i}(t)\right)+C f_{i}\left(x_{i}(t-\tau)\right)+\sum_{j=1}^{N} G_{i j} \Gamma x_{j}(t)+J,
$$

where $i=1,2,3,4, \tau=1, \alpha=0.98, f_{i}\left(x_{i}(t)\right)=\tanh \left(x_{i}(t)\right)$, taking the networks with four nodes and two neurons in every subnetwork. In more detail, the parameters of subnet- 


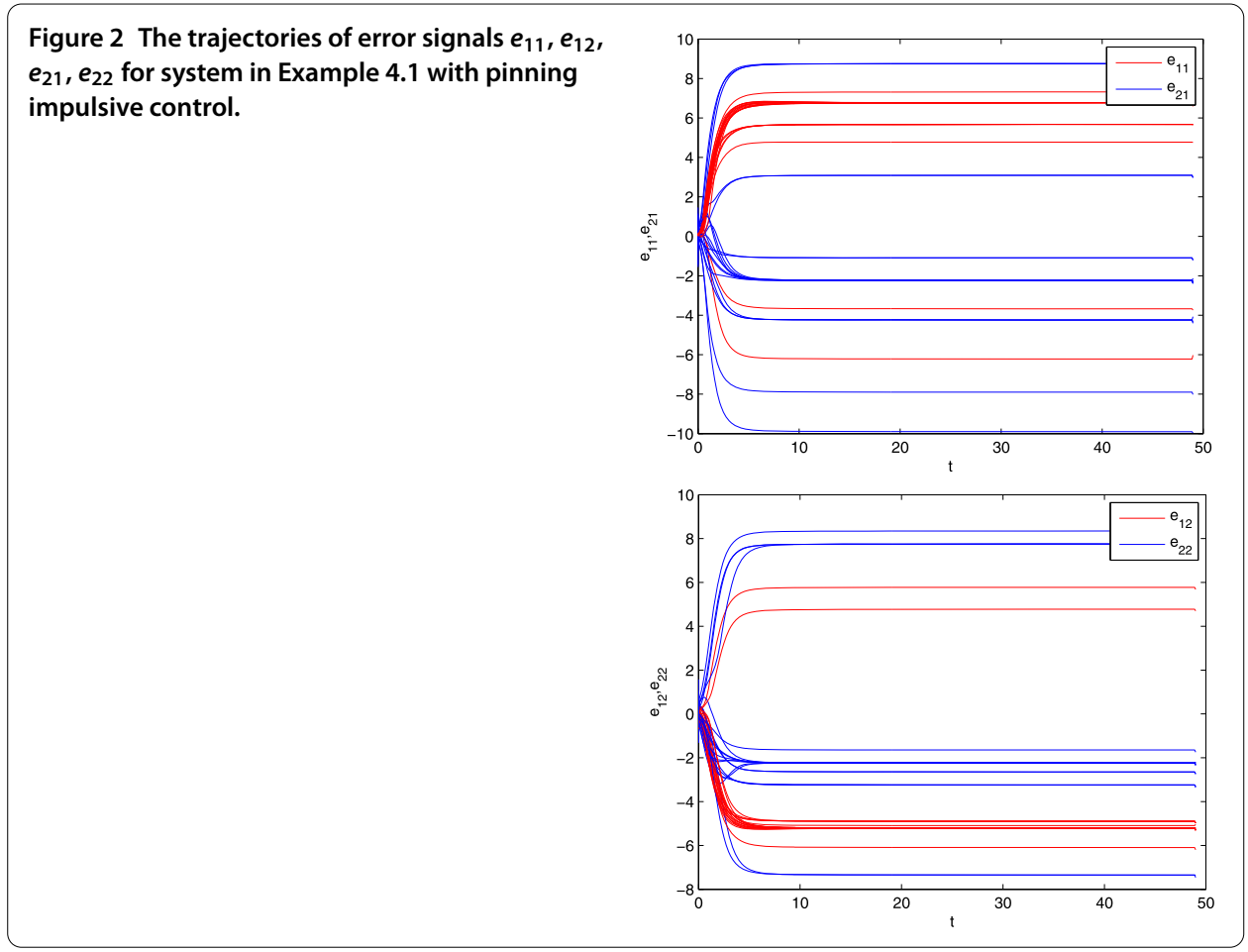

work are given as

$$
\begin{aligned}
A & =\left(\begin{array}{ll}
1 & 0 \\
0 & 1
\end{array}\right), \quad B=\left(\begin{array}{cc}
5 & -1 \\
-0.5 & 4
\end{array}\right), \quad C=\left(\begin{array}{cc}
0.5 & 0 \\
0 & 1
\end{array}\right), \quad J=\left(\begin{array}{l}
0 \\
0
\end{array}\right) \\
G & =\left(\begin{array}{cccc}
1 & 0 & 0 & -0.3 \\
-0.1 & 0.5 & 0 & 0 \\
0 & 0 & 3.1 & 0 \\
0 & 0 & -1 & -4
\end{array}\right) .
\end{aligned}
$$

Let $m=4$ and $\tau=1$. Four shared reference trajectories can be expressed as

$$
{ }^{c} D_{t_{0}, t}^{\alpha} s_{k}(t)=-A s_{k}(t)+B f_{k}\left(s_{k}(t)\right)+C f_{k}\left(s_{k}(t-1)\right)+J, \quad k=1,2,3,4
$$

where $f_{k}\left(s_{k}(t)\right)=\tanh \left(s_{k}(t)\right), k=1,2,3,4, \alpha=0.98$,

$$
A=\left(\begin{array}{ll}
1 & 0 \\
0 & 1
\end{array}\right), \quad B=\left(\begin{array}{cc}
5 & -1 \\
-0.5 & 4
\end{array}\right), \quad C=\left(\begin{array}{cc}
0.5 & 0 \\
0 & 1
\end{array}\right), \quad J=\left(\begin{array}{l}
0 \\
0
\end{array}\right)
$$

We choose $\Gamma=\operatorname{diag}(1,1)$, impulsive gain $\theta_{1}=-0.1, \theta_{2}=-0.5, \rho_{1}=0.5, \rho_{2}=0.4, \eta_{1}=0.05$, $\eta_{2}=0.3$. We can verify that conditions (16)-(19) in Theorem 3.1 hold. By exploiting the 


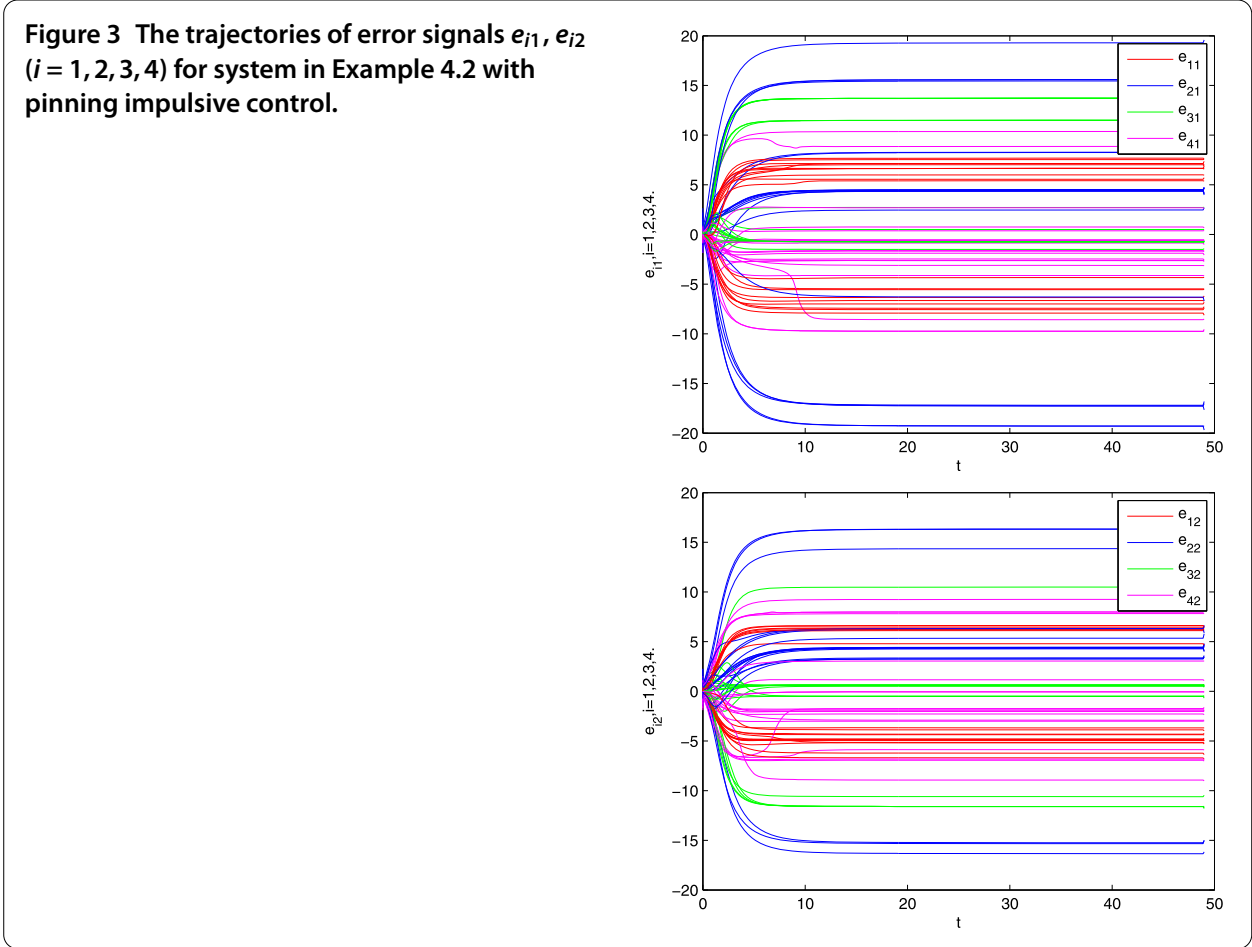

MATLAB LMI Toolbox we get the matrices

$$
\begin{array}{ll}
P & =\left(\begin{array}{ll}
1.0017 & 0.0022 \\
0.0022 & 2.3164
\end{array}\right), \quad \Sigma_{1}=\left(\begin{array}{cc}
1.0023 & 0.1102 \\
0.1102 & 23.0847
\end{array}\right) \\
\Sigma_{2} & =\left(\begin{array}{ll}
1.4908 & 0.0051 \\
0.0051 & 0.0362
\end{array}\right),
\end{array}
$$

and $\frac{\lambda_{\min }(P)}{\lambda_{\max }(P)}=0.8, \sigma_{1}=0.01, \sigma_{2}=0.21$. Set the pinning ratio $\eta_{k}=0.5$. Then system (34) can achieve multi-quasi-synchronization, and Figure 3 depicts the simulation results with 20 random initial values.

Example 4.3 Consider Chua's circuit system as an isolated node of the dynamical network, which is described as follows:

$$
\begin{aligned}
& D_{t_{0}, t}^{\alpha} x_{1}(t)=\varsigma_{0}\left(x_{2}(t)-\varpi_{1} x_{1}(t)-g\left(x_{1}(t)\right)\right), \\
& D_{t_{0}, t}^{\alpha} x_{2}(t)=x_{1}(t)-x_{2}(t)+x_{3}(t), \\
& D_{t_{0}, t}^{\alpha} x_{3}(t)=-\varsigma_{1} x_{2}(t),
\end{aligned}
$$

with $\alpha=0.98, t \geq t_{0}$, the nonlinear function

$$
g\left(x_{1}(t)\right)=\frac{1}{2}\left(\varpi_{1}-\varpi_{0}\right)\left(\left|x_{1}(t)+1\right|-\left|x_{1}(t)-1\right|\right),
$$

and the parameters $\varpi_{0}=-\frac{1}{7}, \varpi_{1}=\frac{2}{7}, \varsigma_{0}=9$. This system exhibits chaotic behavior, as given in Figure 4. System (35) can be represented as the error system (5) consisting of four 


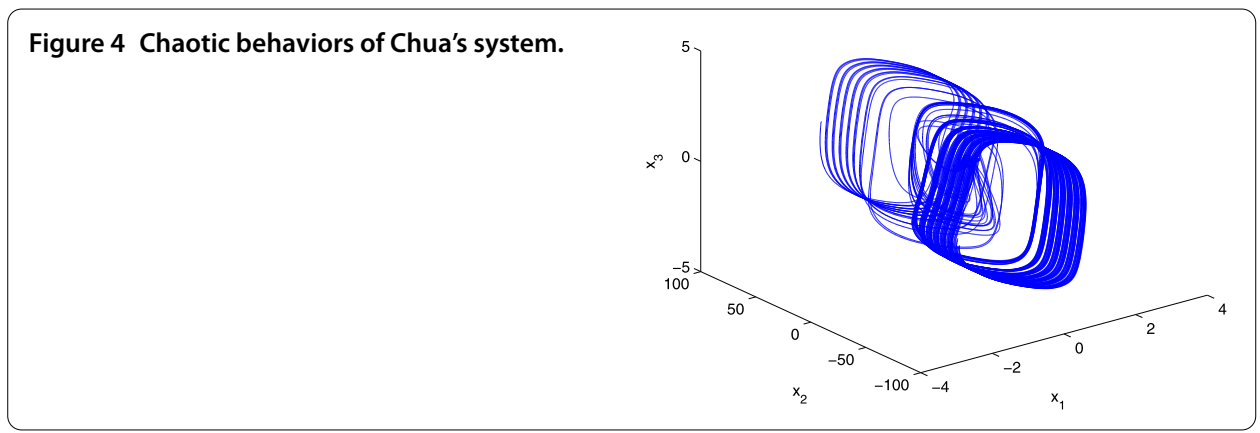

nodes $(N=4)$ with parameters

$$
\begin{aligned}
A & =\left(\begin{array}{ccc}
-\varpi_{0} \varsigma_{0} & \varsigma_{0} & 0 \\
1 & -1 & 1 \\
0 & -\varsigma_{1} & 0
\end{array}\right), \quad B=\left(\begin{array}{ccc}
1 & 0 & 0 \\
-1 & 0 & 0 \\
2 & 0 & -1
\end{array}\right), \quad C=\left(\begin{array}{ccc}
\varpi_{1}-\varpi_{0} & 0 & 0 \\
-1 & 1 & 0 \\
\varpi_{1} & 0 & 0
\end{array}\right), \\
G & =\left(\begin{array}{cccc}
-1 & 0.5 & 0.3 & -1.3 \\
1 & -0.5 & 3 & 0 \\
0 & 1 & 3.1 & 1 \\
-3 & 0 & -1 & -1.5
\end{array}\right), \quad J=\left(\begin{array}{l}
0 \\
0 \\
0
\end{array}\right),
\end{aligned}
$$

$f_{i}\left(x_{i}(t)\right)=\frac{1}{2}\left(\left|x_{i}(t)+1\right|-\left|x_{i}(t)-1\right|\right), \Gamma=\operatorname{diag}\{1,1,1\}$, impulsive gain $\theta_{1}=\theta_{2}=\theta_{3}=0.1, \rho_{1}=$ $\rho_{2}=\rho_{3}=0.4, \eta_{1}=\eta_{2}=\eta_{3}=0.03$. Let nodes 1,2 , and 4 be pinning controlled nodes. By Theorem 3.1 we get the matrices

$$
\begin{aligned}
P & =\left(\begin{array}{lll}
1.0017 & 0.0098 & 1.0761 \\
0.0314 & 2.3164 & 0.0091 \\
0.085 & 0.6316 & 1.3141
\end{array}\right), \\
\Sigma_{1} & =\left(\begin{array}{lll}
1.0023 & 0.0076 & 0.3211 \\
0.0091 & 3.0034 & 0.7102 \\
0.0012 & 0.0031 & 11.23207
\end{array}\right), \\
\Sigma_{2} & =\left(\begin{array}{lll}
1.4908 & 0.0014 & 0.3908 \\
0.0016 & 1.0908 & 0.0012 \\
0.6601 & 0.0076 & 0.0316
\end{array}\right) .
\end{aligned}
$$

The state trajectories of multi-quasi-synchronization errors $e_{i 1}(t), e_{i 2}(t)$, and $e_{i 3}(t)(i=$ $1,2,3,4)$ with 20 random initial values are depicted in Figure 5. Based on our analysis, the effectiveness of the designed pinning impulsive control is verified.

\section{Concluding remarks}

In this paper, multi-quasi-synchronization of coupled fractional-order neural networks with delays has been studied by applying pinning impulsive control. For this control strategy, we divide the node set into several disjoint subsets. By using comparison principle and Lyapunov method, several sufficient conditions have been derived to realize multi-quasi-synchronization. In the future, it is very interesting to study the multi-quasisynchronization of coupled complex control systems $[43,44]$. 


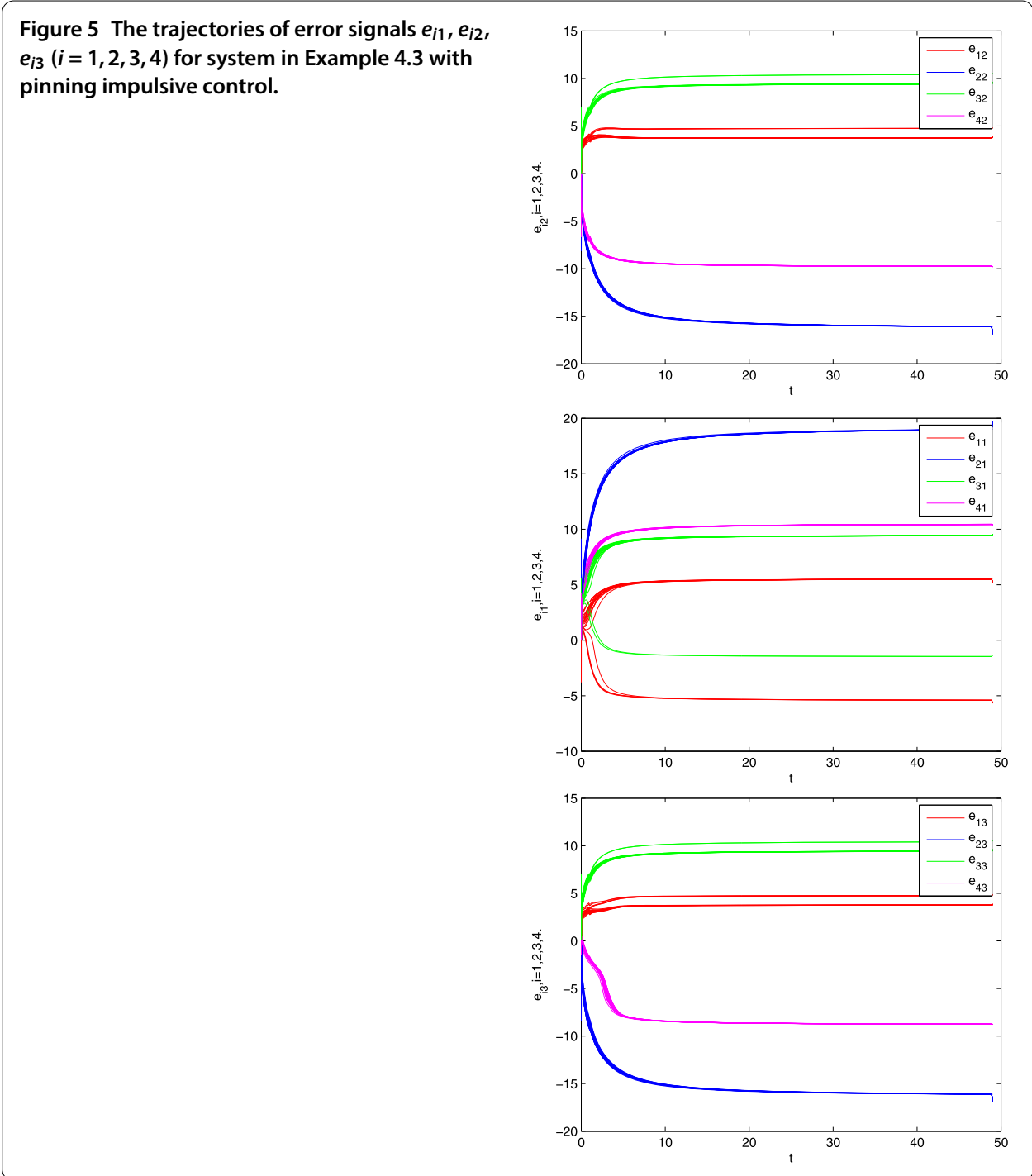

Acknowledgements

The work is supported by the Natural Science Foundation of China under Grants 61640309 and 61773152.

Competing interests

The authors declare that they have no competing interests.

Authors' contributions

Both authors contributed equally to the writing of this paper. Both authors read and approved the final manuscript.

\section{Author details}

${ }^{1}$ College of Mathematics and Statistics, Hubei Normal University, Huangshi, 435002, China. ${ }^{2}$ Institute for Information and System Science, Xi'an Jiaotong University, Xi'an, 710049, China. ${ }^{3}$ School of Automation, Huazhong University of Science and Technology, Wuhan, 430074, China.

\section{Publisher's Note}

Springer Nature remains neutral with regard to jurisdictional claims in published maps and institutional affiliations.

Received: 1 June 2017 Accepted: 31 October 2017 Published online: 09 November 2017

\section{References}

1. Li, CP, Zhang, FR: A survey on the stability of fractional differential equations. Eur. Phys. J. Spec. Top. 193, 27-47 (2011)

2. Koeller, R: Applications of fractional calculus to the theory of viscoelasticity. J. Appl. Mech. 51, 299-307 (1984)

3. Gallegos, JA, Duarte, MA: On the Lyapunov theory for fractional order systems. Appl. Math. Comput. 287-288 $161-170(2016)$ 
4. Wu, AL, Zeng, ZG: Boundedness, Mittag-Leffler stability and asymptotical $\omega$-periodicity of fractional-order fuzzy neural networks. Neural Netw. 74, 73-84 (2016)

5. Wu, AL, Zeng, ZG, Song, XG: Global Mittag-Leffler stabilization of fractional-order bidirectional associative memory neural networks. Neurocomputing 177, 489-496 (2016)

6. Yang, XJ: Fractional derivatives of constant and variable orders applied to anomalous relaxation models in heat-transfer problems. Therm. Sci. 21, 1161-1171 (2017)

7. Yang, XJ, Srivastava, HM, Tenreiro Machado, JA: A new fractional derivative without singular kernel: application to the modelling of the steady heat flow. Therm. Sci. 20, 753-756 (2016)

8. Yang, XJ, Tenreiro Machado, JA: A new fractional operator of variable order: application in the description of anomalous diffusion. Phys. A, Stat. Mech. Appl. 481, 276-283 (2017)

9. Yang, XJ, Tenreiro Machado, JA, Baleanu, D, Cattani, C: On exact traveling-wave solutions for local fractional Korteweg-de Vries equation. Chaos 26, 084312 (2016)

10. Yang, XJ, Tenreiro Machado, JA, Baleanu, D: Exact traveling-wave solution for local fractional Boussinesq equation in fractal domain. Fractals 25, 1740006 (2017)

11. Yang, XJ, Gao, F, Srivastava, HM: Exact travelling wave solutions for the local fractional two-dimensional Burgers-type equations. Appl. Math. Comput. 73, 203-210 (2017)

12. Lu, JG: Chaotic dynamics and synchronization of fractional-order Arneodo's systems. Chaos Solitons Fractals 26 1125-1133 (2005)

13. Yuan, LG, Yang, QG, Wu, RC, Sun, J, Ma, TD: Parameter identification and synchronization of fractional-order chaotic systems. Commun. Nonlinear Sci. Numer. Simul. 17, 305-316 (2012)

14. Wang, GS, Xiao, JW, Wang, YW, Yi, JW: Adaptive pinning cluster synchronization of fractional-order complex dynamical networks. Appl. Math. Comput. 231, 347-356 (2014)

15. Yang, LX, Jiang, J: Adaptive synchronization of drive-response fractional-order complex dynamical networks with uncertain parameters. Commun. Nonlinear Sci. Numer. Simul. 19, 1496-1506 (2014)

16. Wong, WK, Li, HJ, Leung, SYS: Robust synchronization of fractional-order complex dynamical networks with parametric uncertainties. Commun. Nonlinear Sci. Numer. Simul. 17, 4877-4890 (2012)

17. Bao, HB, Cao, JD: Projective synchronization of fractional-order memristor-based neural networks. Neural Netw. 63, 1-9 (2015)

18. Velmurugan, G, Rakkiyappan, R: Hybrid projective synchronization of fractional-order memristor-based neural networks with time delays. Nonlinear Dyn. 83, 419-432 (2016)

19. Chen, JJ, Zeng, ZG, Jiang, P: Global Mittag-Leffler stability and synchronization of memristor-based fractional-order neural networks. Neural Netw. 51, 1-8 (2014)

20. Ma, TD, Zhang, J: Hybrid synchronization of coupled fractional-order complex networks. Neurocomputing 157, 166-172 (2015)

21. Ma, TD, Zhang, J: Adaptive hybrid projective synchronization of two coupled fractional-order complex networks with different sizes. Neurocomputing 164, 182-189 (2015)

22. Giresse, TA, Crepin, KT: Chaos generalized synchronization of coupled Mathieu-Van der Pol and coupled Duffing-Van der Pol systems using fractional order derivative. Chaos Solitons Fractals 98, 88-100 (2017)

23. Wu, GC, Baleanu, D, Xie, HP, Chen, FL: Chaos synchronization of fractional chaotic maps based on the stability condition. Phys. A, Stat. Mech. Appl. 460, 374-383 (2016)

24. Yu, WW, Chen, GR, Lu, JH: On pinning synchronization of complex dynamical networks. Automatica 45, 429-435 (2009)

25. Wang, T, Ding, YS, Zhang, LK, Hao, R: Adaptive feedback synchronisation of complex dynamical network with discrete-time communications and delayed nodes. Int. J. Syst. Sci. 47, 2563-2571 (2016)

26. Zhang, W, Tang, Y, Miao, Q, Fang, JA: Synchronization of stochastic dynamical networks under impulsive control with time delays. IEEE Trans. Neural Netw. Learn. Syst. 25, 1758-1768 (2013)

27. Sun, W, Hu, TS, Chen, Z: Impulsive synchronization of a general nonlinear coupled complex network. Commun. Nonlinear Sci. Numer. Simul. 16, 4501-4507 (2011)

28. Jajarmi, A, Hajipour, M, Baleanu, D: New aspects of the adaptive synchronization and hyperchaos suppression of a financial model. Chaos Solitons Fractals 99, 285-296 (2017)

29. Xia, WG, Cao, JD: Pinning synchronization of delayed dynamical networks via periodically intermittent control. Chaos 19,013120 (2009)

30. Liu, B, Liu, XZ, Chen, GR, Wang, HY: Robust impulsive synchronization of uncertain dynamical networks. IEEE Trans. Circuits Syst. I, Regul. Pap. 52, 1431-1441 (2005)

31. Song, Q, Cao, JD: On pinning synchronization of directed and undirected complex dynamical networks. IEEE Trans. Circuits Syst. I, Regul. Pap. 57, 672-680 (2010)

32. Wang, YL, Cao, JD, Hu, JQ: Stochastic synchronization of coupled delayed neural networks with switching topologies via single pinning impulsive control. Neural Comput. Appl. 26, 1739-1749 (2015)

33. He, WL, Qian, F, Cao, JD: Pinning-controlled synchronization of delayed neural networks with distributed-delay coupling via impulsive control. Neural Netw. 85, 1-9 (2017)

34. He, DX, Ling, G, Guan, ZH, Hu, B, Liao, RQ: Multisynchronization of coupled heterogeneous genetic oscillator networks via partial impulsive control. IEEE Trans. Neural Netw. Learn. Syst. (2016). doi:10.1109/TNNLS.2016.2619907

35. Wang, YW, Yang, W, Xiao, JW, Zeng, ZG: Impulsive multisynchronization of coupled multistable neural networks with time-varying delay. IEEE Trans. Neural Netw. Learn. Syst. 28, 1560-1571 (2017)

36. Liu, XY, Chen, TP, Cao, JD, Lu, WL: Dissipativity and quasi-synchronization for neural networks with discontinuous activations and parameter mismatches. Neural Netw. 24, 1013-1021 (2011)

37. Duarte-Mermoud, M, Aguila-Camacho, N, Gallegos, JA, Castro-Linares, R: Using general quadratic Lyapunov functions to prove Lyapunov uniform stability for fractional order systems. Commun. Nonlinear Sci. Numer. Simul. 22, 650-659 (2015)

38. Pan, LJ, Cao, JD: Stochastic quasi-synchronization for delayed dynamical networks via intermittent control. Commun. Nonlinear Sci. Numer. Simul. 17, 1332-1343 (2012)

39. Wang, LM, Song, QK, Liu, YR, Zhao, ZJ, Alsaadi, FE: Global asymptotic stability of impulsive fractional-order complex-valued neural networks with time delay. Neurocomputing 243, 49-59 (2017) 
40. Morgado, ML, Ford, NJ, Lima, PM: Analysis and numerical methods for fractional differential equations with delay. J. Comput. Appl. Math. 252, 159-168 (2013)

41. Miller, KS, Samko, SG: Completely monotonic functions. Integral Transforms Spec. Funct. 12, 389-402 (2001)

42. Deng, $\mathrm{WH}, \mathrm{Li}, \mathrm{CP}, \mathrm{Lü}, \mathrm{JH}$ : Stability analysis of linear fractional differential system with multiple time delays. Nonlinear Dyn. 48, 409-416 (2007)

43. Lin, D, Wang, XY, Nian, FZ, Zhang, YL: Dynamic fuzzy neural networks modeling and adaptive backstepping tracking control of uncertain chaotic systems. Neurocomputing 73, 2873-2881 (2010)

44. Li, HY, Chen, ZR, Wu, LG, Lam, HK, Du, HP: Event-triggered fault detection of nonlinear networked systems. IEEE Trans. Cybern. 47, 1041-1052 (2017)

Submit your manuscript to a SpringerOpen ${ }^{\circ}$ journal and benefit from:

- Convenient online submission

$\checkmark$ Rigorous peer review

- Open access: articles freely available online

- High visibility within the field

- Retaining the copyright to your article

Submit your next manuscript at $\gg$ springeropen.com 